\section{Thickening and Contrast Enhancement of Spinal Roots on MR Imaging in Guillain-Barré Syndrome: Thoughts on Pathologic Background}

I read with interest the article by Zuccoli et al $^{1}$ evaluating the MR imaging findings in 17 children affected by Guillain-Barré syndrome (GBS) or Miller Fisher syndrome. Fourteen patients demonstrated cauda equina enhancement, with predominant involvement of the anterior roots. Furthermore, isolated enhancement of the anterior spinal roots was observed in 3 patients. Given that the authors make no reference to the pathologic hallmark of such neuroimaging findings, I wish to comment on this question.

In 4 detailed clinicopathologic studies in GBS, including complete transverse semithin sections of lumbosacral roots, we found constant but variable radicular lesions ranging from widespread inflammatory demyelination to scattered inflammatory infiltrates. ${ }^{2-5}$ In 1 study from a patient presenting with pure motor GBS, inflammatory demyelination with secondary axonal degeneration was restricted to ventral spinal roots. ${ }^{2}$ Figure 1 illustrates typical findings in the L5 ventral root. Considering the constancy of radicular inflammation, the reported MR imaging findings are not unexpected in any form of GBS. Absence of an epi-perineurium in the preforaminal spinal roots and, by extension, in the cranial nerves in their subarachnoid trajectory accounts for the observed thickening on MR imaging. External to the subarachnoid angle, the peripheral nerve trunks possess epi-perineurium, which is relatively inelastic, thus not allowing any significant increase of their diameters. Precise inflammatory demyelination of these nerve trunks may imply an increase of endoneurial fluid nerve pressure potentially causing endoneurial ischemia and wallerian-like degeneration. ${ }^{3-5}$

The predominance of inflammatory lesions in spinal roots and particularly in the ventral ones is an outstanding feature in GBS and in experimental allergic neuritis. Antigen differences between motor and sensory neurons probably account for greater and selective motor involvement. Moreover, it could be pathogenetically relevant that the richest source of $\mathrm{P}_{2}$, the major antigen for producing experimental allergic neuritis, is in the ventral roots of the cauda equina. ${ }^{2}$ Finally, predominance of lesions in spinal roots may be correlated with their less efficient blood-nerve barrier compared with more distal nerve trunks.

\section{References}

1. Zuccoli G, Panigrahy A, Bailey A, et al. Redefining the Guillain-Barré spectrum in children: neuroimaging findings of cranial nerve involvement. AJNR Am J Neuroradiol 2011;32:639-42

2. Berciano J, Coria F, Montón F, et al. Axonal form of Guillain-Barré syndrome: evidence for macrophage-associated demyelination. Muscle Nerve 1993;16: $744-51$

3. Berciano J, Figols J, García A, et al. Fulminant Guillain-Barré syndrome with universal inexcitability of peripheral nerves: a clinicopathological study. Muscle Nerve 1997;20:846-57

4. Berciano J, García A, Figols J, et al. Perineurium contributes to axonal damage in acute inflammatory demyelinating polyneuropathy. Neurology 2000;55: $552-59$

5. Berciano J, García A, Villagrá NT, et al. Severe Guillain-Barré syndrome: sorting out the pathological hallmark in an electrophysiological axonal case. $J$ Peripher Nerv Syst 2009;14:54-63

J. Berciano Service of Neurology University Hospital "Marqués de Vadecilla" (UC and CIBERNED) Santander, Spain
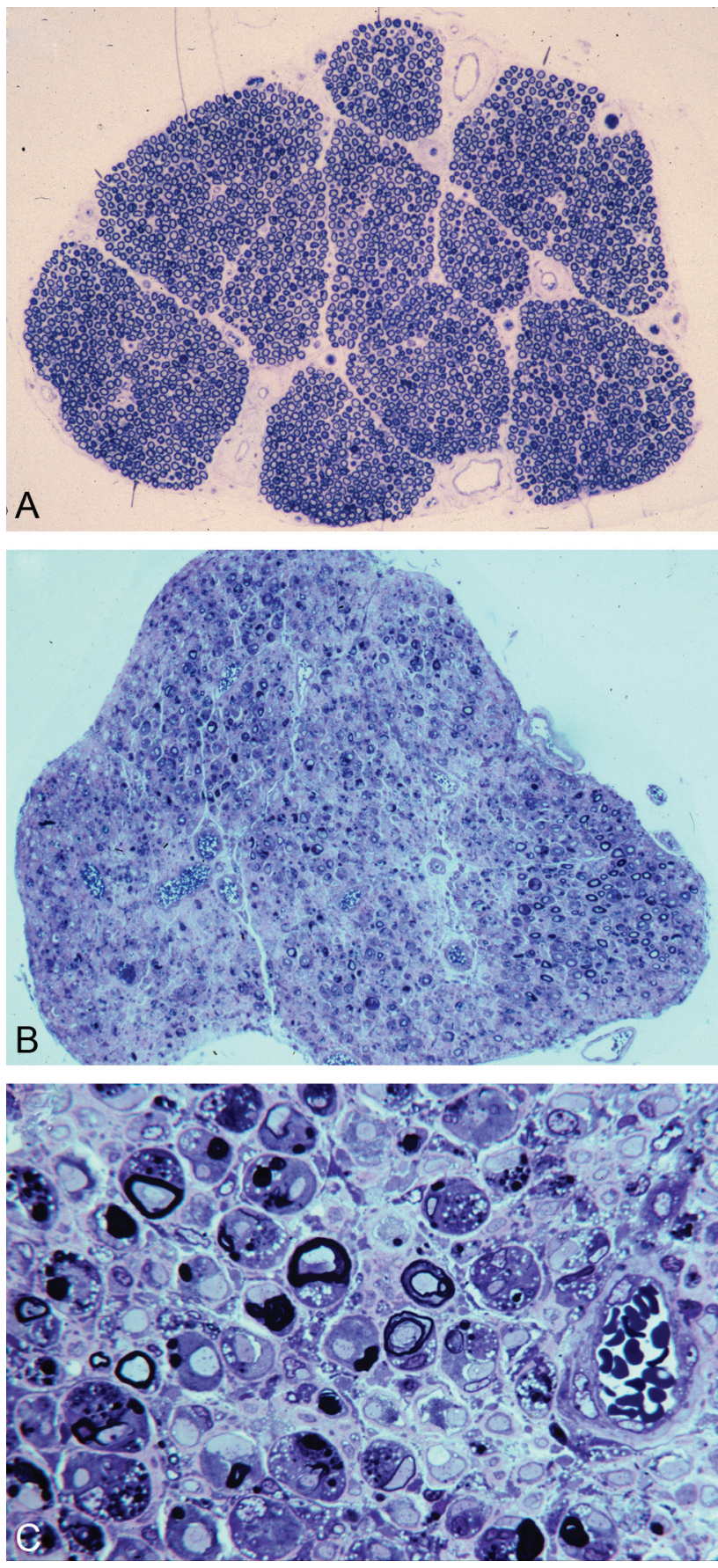

Fig 1. $A$, Complete semithin transverse section of the $L 5$ ventral root in a control subject with no pathology. $B$, Complete semithin transverse section of the $L 5$ ventral root from the patient reported in reference 3 . Note that despite apparent severe reduction of myelinated fibers, the transverse fascicular area is preserved or even increased $(A$ and $B$, toluidine blue, original magnification, $\times 10$ objective). $C$, At higher magnification, note extensive inflammatory demyelination with numerous denuded axons and foamy laden macrophages (toluidine blue, original magnification, $\times 63$ oil immersion objective). 